\title{
THE EXPERIMENTAL PATHOGENICITY IN MICE OF STRAINS OF PROTEUS OF ANIMAL ORIGIN
}

\author{
By J. E. PHILLIPS \\ Department of Veterinary Bacteriology, Royal (Dick) School \\ of Veterinary Studies, University of Edinburgh
}

(With Plates 8 and 9)

\begin{abstract}
Phillips (1955) examined 214 Proteus strains of animal origin and found that their in vitro properties corresponded closely with those of strains derived from man. The pathogenic properties of this group of bacteria in experimental animals, particularly in mice, have not been studied very fully, and the published results show a considerable divergence of opinion (Havens \& Ridgeway, 1929; Lovell, 1929; Yacob, 1932; Lasserre, Velu \& Soulie, 1941 ; Lesbouyries, 1942). The inconsistent findings may have been due, in part, to the failure of these authors to estimate the dose of viable organisms used.

It is the purpose of this paper to describe the pathogenic effects in mice of a number of strains of Proteus recovered from animals.
\end{abstract}

\section{TECHNICAL METHODS}

Fifty-seven Proteus strains of the above collection (Phillips, 1955) were used, together with ten strains from the National Collection of Type Cultures. Most of the animal strains had been subcultured for 4-5 years but twelve strains had been subcultured less than three times.

Mice were inoculated either with $18 \mathrm{hr}$. nutrient broth cultures or with suspensions of cultures grown for $\mathbf{1 8} \mathrm{hr}$. on nutrient agar and standardized in sterile normal saline to Brown's opacity tube no. 4. All strains were inoculated intravenously, and some were also given by the intraperitoneal and subcutaneous routes.

Viable counts were made by the roll tube method using nutrient agar containing $0.5 \%$ sodium taurocholate. This medium inhibited swarming sufficiently for an accurate estimation of the number of viable organisms to be made.

\section{RESULTS}

Preliminary experiments, using two newly isolated strains, suggested that Proteus was non-pathogenic for mice.

Further work, however, using two other recently isolated strains in doses of $7.5 \times 10^{8}$ and $7.5 \times 10^{7}$ viable organisms, showed a pathogenic effect in which mice inoculated with the larger dose died within $24 \mathrm{hr}$. with septicaemia. Those receiving the smaller dose survived for periods varying between 19 and 34 days when they either died or were killed for post-mortem examination. At autopsy these 
showed lesions in the kidneys consisting of circular yellow areas projecting slightly above the surface of the organ (Pl. 8), while macroscopic section showed that the lesion extended to the pelvis; Proteus was isolated from these in every case. No lesions were found in any other organ.

The effects of inoculating living and dead organisms were compared and each of five further strains of Proteus was inoculated into two groups of five mice; one group received living cells (approximately $7 \cdot 5 \times 10^{7}$ viable organisms) and the other group an equal amount of bacterial suspension which had been heated at $100^{\circ} \mathrm{C}$. for $30 \mathrm{~min}$. and was subsequently shown to be sterile. One mouse from each group was killed at intervals of 7 days and post-mortem and cultural examinations made. Each kidney was also examined histologically. The results are shown in Table 1. Although the living suspensions of Proteus did not induce renal lesions in every mouse, such lesions were observed macroscopically in at least two animals with each strain. Cultures were obtained from all kidneys showing lesions, but in some animals Proteus was also recovered from an apparently normal kidney. None of the mice inoculated with killed cultures developed renal lesions, none showed microscopical abnormalities and no bacteria were recovered in culture.

Table 1. Number of mice showing renal lesions after administration of living and killed cultures of Proteus

\begin{tabular}{|c|c|c|c|c|c|c|c|c|}
\hline \multirow{2}{*}{ Strain no. } & \multicolumn{4}{|c|}{ Living cultures } & \multicolumn{4}{|c|}{ Killed cultures } \\
\hline & $\mathrm{N}$ & L & M & $\mathbf{P}$ & $\mathrm{N}$ & L & M & $\mathbf{P}$ \\
\hline 230 & 5 & 2 & 2 & 2 & 5 & 0 & 0 & 0 \\
\hline 232 & 5 & 2 & 3 & 3 & 5 & 0 & 0 & 0 \\
\hline 233 & 5 & 2 & 2 & 2 & $\mathbf{5}$ & 0 & 0 & 0 \\
\hline 234 & $\mathbf{5}$ & 3 & 3 & 4 & 5 & 0 & 0 & 0 \\
\hline 235 & $\mathbf{5}$ & 2 & 3 & 3 & 5 & 0 & 0 & 0 \\
\hline
\end{tabular}

$\mathrm{N}=$ number of mice examined. $\mathrm{L}=$ number of mice showing macroscopic lesions in the kidneys. $\mathrm{M}=$ number of mice showing microscopic lesions in the kidneys. $\mathrm{P}=$ number of mice from the kidneys of which Proteus was recovered.

In those kidneys showing definite macroscopic lesions there were considerable and usually extensive histological changes consisting of tubular degeneration and necrosis which sometimes extended to involve the glomeruli; in some cases these structures were unaffected although surrounded by an intense cellular reaction. The lumina of the tubules were packed with masses of Gram-negative organisms. The changes in the tubules extended from the cortex, through the medulla, to the pelvis and gave the appearance of a pyelonephritis. In some of the older lesions, in mice killed later than 3 weeks after inoculation, less marked histological changes were present, consisting of an interstitial fibrosis suggesting healing.

The inoculation of other strains of Proteus into mice showed that renal lesions appeared only when the organisms were administered by the intravenous route. It was also found that large doses tended to produce a septicaemia, while very small doses were completely non-pathogenic.

The sequence of events occurring in the kidney when suspensions of Proteus are 
inoculated intravenously into mice was studied by inoculating fifty mice each with $7.5 \times 10^{7}$ viable organisms of a strain which was known to produce kidney lesions, and thereafter killing five animals daily for post-mortem, cultural and histological examination of the kidneys. The results are given in Table 2 which shows that macroscopic changes were present within 3 days and consisted of a slight enlargement of the organ which was paler than normal. From the fourth day until the end of the experiment, well-marked necrotic lesions were found. Proteus was isolated from the kidneys each day and, in the latter part of the experiment, from a number of kidneys with no macroscopic lesions, showing that Proteus can persist in the tissues for at least 10 days.

Table 2. Number of mice showing kidney lesions and positive kidney cultures at varying intervals after the administration of Proteus

$\begin{array}{crrrr}\begin{array}{c}\text { Days after } \\ \text { inoculation }\end{array} & \text { N } & \text { L } & \text { M } & \text { P } \\ 1 & 5 & 0 & 0 & 5 \\ 2 & 5 & 0 & 2 & 5 \\ 3 & 5 & 2 & 3 & 2 \\ 4 & 5 & 3 & 4 & 3 \\ 5 & 5 & 3 & 3 & 3 \\ 6 & 5 & 2 & 2 & 2 \\ 7 & 5 & 5 & 5 & 4 \\ 8 & 5 & 2 & 3 & 1 \\ 9 & 5 & 2 & 3 & 3 \\ 10 & 5 & 1 & 2 & 3 \\ \text { Total } & 50 & 20 & 27 & 31\end{array}$

$\mathrm{N}=$ number of mice examined. $\mathrm{L}=$ number of mice showing macroscopic lesions in the kidneys. $M=$ number of mice showing microscopic lesions in the kidneys. $P=$ number of mice from the kidneys of which Proteus was recovered.

Microscopic examination of the kidneys showed histological changes in a number of cases where no naked-eye change was apparent. The earliest change, seen on the second day, consisted of an infiltration of leucocytes in several areas in the medulla and to a lesser extent in the cortex, and, in one case, leucocytes were observed in the pelvis of the kidney (Pl. 9, fig. 1). On the following day leucocytic infiltration was still seen and degeneration of the cells of the uriniferous tubules had commenced. By the fourth day tubular degeneration had advanced and large masses of organisms were seen lying within the lumina of the tubules, while the number of leucocytes had increased considerably and many were present in the pelvis. At this stage the lesion had extended to involve the cortex. On subsequent days necrosis of the renal tubules was seen, the lesion consisting of a central mass of necrotic renal cells, together with large numbers of bacteria, surrounded by an extensive zone of leucocytes (Pl. 9, fig. 2). Varying amounts of kidney damage were observed, and in some cases the lesions were so extensive as to involve the whole organ.

Forty-five strains of Proteus of animal origin and ten N.C.T.C. strains were inoculated intravenously in doses of $15 \times 10^{7}$ viable organisms into each of five 
mice and these were killed 7 days later. Four animal strains of Proteus did not produce kidney lesions, and the results of cultural examinations indicated that they were eliminated from the kidney tissue within 7 days. Eighteen strains persisted in the kidney throughout the time of the experiment, although they did not produce any macroscopic lesions. Twenty-three strains induced lesions and persisted in the kidney tissue; of the National Collection of Type Culture strains two only produced macroscopic lesions. These results show that differences in pathogenicity exist.

\section{DISCUSSION}

Although there appears to be no previous record of renal lesions in mice caused by Proteus, the phenomenon is well recognized with staphylococci (Gorrill, 1951), Corynebacterium renale (Lovell \& Cotchin, 1946) and Pseudomonas pyocyanea (Gorrill, 1952). The renal lesions might be due to bacterial cells causing embolism of the renal capillaries, but the results with the killed suspensions of Proteus do not uphold this view. This and the absence of lesions in any other organs where embolism might occur suggest that the localization of Proteus in the kidney is the expression of some selectivity on the part of these organisms. Lovell (1951) found that Corynebacterium renale settled in the medulla of the kidney after intravenous inoculation into mice, producing lesions which later extended to involve the cortex. He believed that this distribution of the lesions was associated with and possibly dependent upon the production of urease by this organism. Proteus organisms are very active urease producers, and the distribution of lesions in the kidney suggests that the organisms settle near the renal tubules where the concentration of urea is highest. Perhaps damage to the renal tissue is the result of an increase in $\mathrm{pH}$, brought about by decomposition of urea, which favours growth of the organisms. A similar mechanism might account for the lesions described by Gorrill (1952) with Pseudomonas pyocyanea which also produces urease, although less actively than Proteus. However, urease production is probably not entirely responsible for localization in the kidney, since several typical strains of Proteus did not produce renal lesions and, moreover, were unable to persist for any length of time in the kidney tissue.

Variable results like those obtained with Proteus, depending upon the size of the dose, namely septicaemic death or renal lesions, were observed by Gorrill (1952) with Pseudomonas pyocyanea, and he was not able to produce renal lesions with any certainty by the intraperitoneal route. Miles (1951) demonstrated the septicaemic effect of Proteus vulgaris by intraperitoneal inoculation, but did not record kidney lesions.

The variation in pathogenicity of Proteus strains observed during this work agrees with the observation of earlier workers. In spite of laboratory culture for 4-5 years many of the strains of animal origin were able to produce renal lesions, although the National Collection of Type Culture strains, which may have been grown on artificial media for many years, were less active in this respect. 


\section{SUMMARY}

1. Strains of Proteus derived from animals produced septicaemia in mice when given in large doses intravenously, but smaller doses caused necrotic lesions in the kidneys. The organisms failed to produce kidney lesions when administered intraperitoneally or subcutaneously but did cause septicaemia.

2. Killed suspensions of Proteus failed to induce kidney lesions.

3. Strain differences in pathogenicity were found, some strains producing nakedeye lesions in the kidney, others giving microscopic changes only in this organ; of those strains which caused no changes in the kidney tissue, some persisted for several days and others were eliminated rapidly.

4. The mechanism of this localization in the kidney is discussed.

The writer wishes to thank Dr A. Wilson Taylor and Prof. R. Lovell for their advice and helpful suggestions and Mr R. Hood for the photographs.

\section{REFERENCES}

GoRrILL, R. H. (1951). Brit. J. exp. Path. 32, 151.

GoRrILl, R. H. (1952). J. Path. Bact. 64, 857.

Havens, L. C. \& Ridgeway, C. (1929). J. prev. Med. 3, 159.

Lasserre, R., Velu, H. \& Soulie, P. (1941). Bull. Acad. vét. Fr. 14, 83.

Lesbouyries, M. (1942). Bull. Acad. vét. Fr. 15, 278.

Lovelt, R. (1929). J. Path. Bact. 32, 79.

LOVELt, R. (1951). Vet. Rec. 63, 645.

Lovelt, R. \& Cotchin, E. (1946). J. comp. Path. 56, 205.

Miles, A. A. (1951). J. gen. Microbiol. 5, 307.

Phillirs, J. E. (1955). J. Hyg., Camb., 53.

YАСов, M. (1932). Indian J. med. Res. 19, 787.

\section{EXPLANATION OF PLATES}

\section{Plate 8}

Plate 1 : Kidney lesions in a mouse 19 days after the intravenous inoculation of $7.5 \times 10^{7}$ viable Proteus organisms.

\section{Plate 9}

Fig. 1. Section of kidney of a mouse 2 days after the intravenous inoculation of $7.5 \times 10^{7}$ viable Proteus organisms, showing infiltration of leucocytes into the medulla and pelvis $(\times 130)$.

Fig. 2. Section of kidney of a mouse 5 days after the intravenous inoculation of $7.5 \times 10^{7}$ viable Proteus organisms, showing extensive necrosis of the medulla and cortex with leucocytic infiltration $(\times 167)$.

(MS. received for publication 31. xII. 54) 


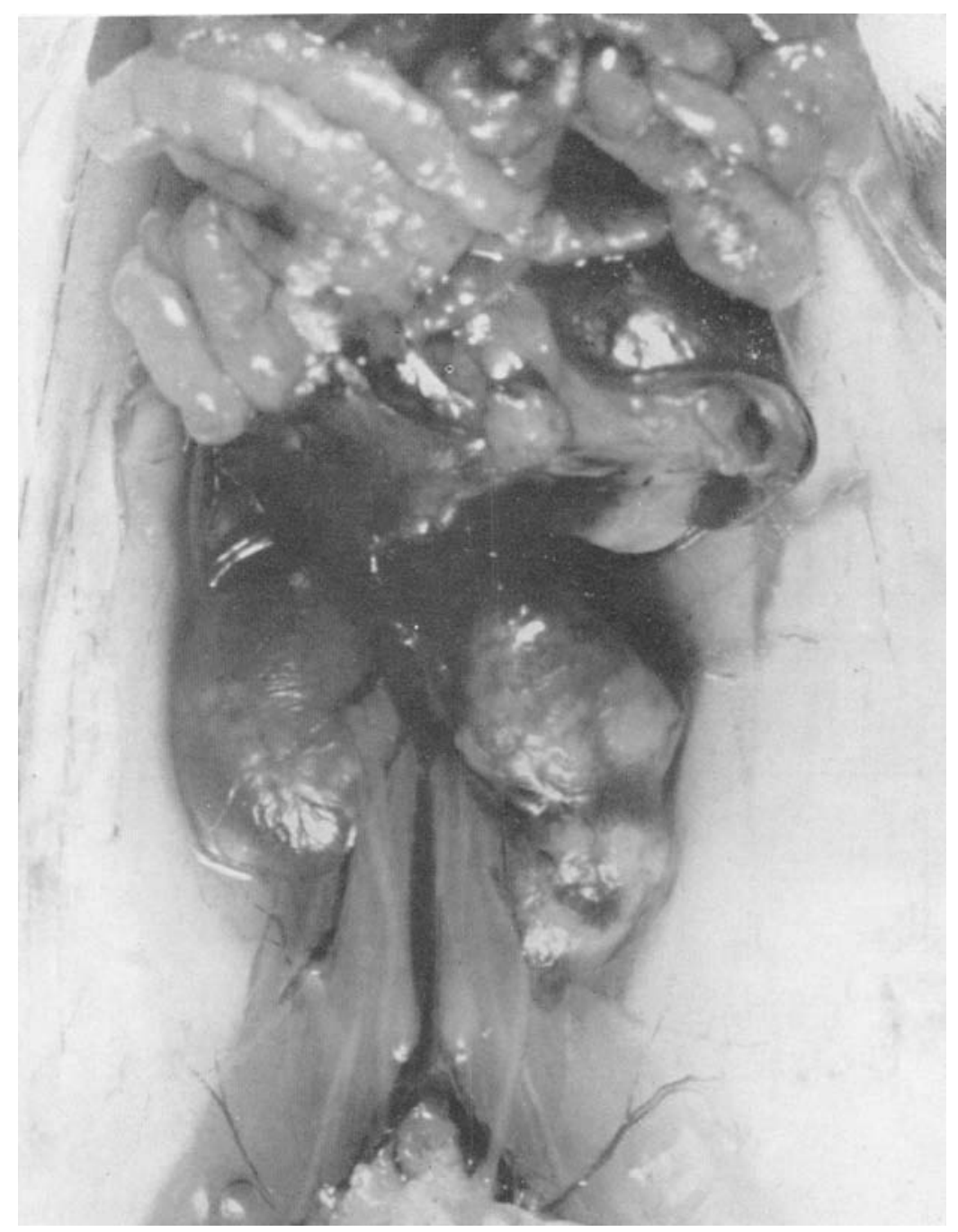



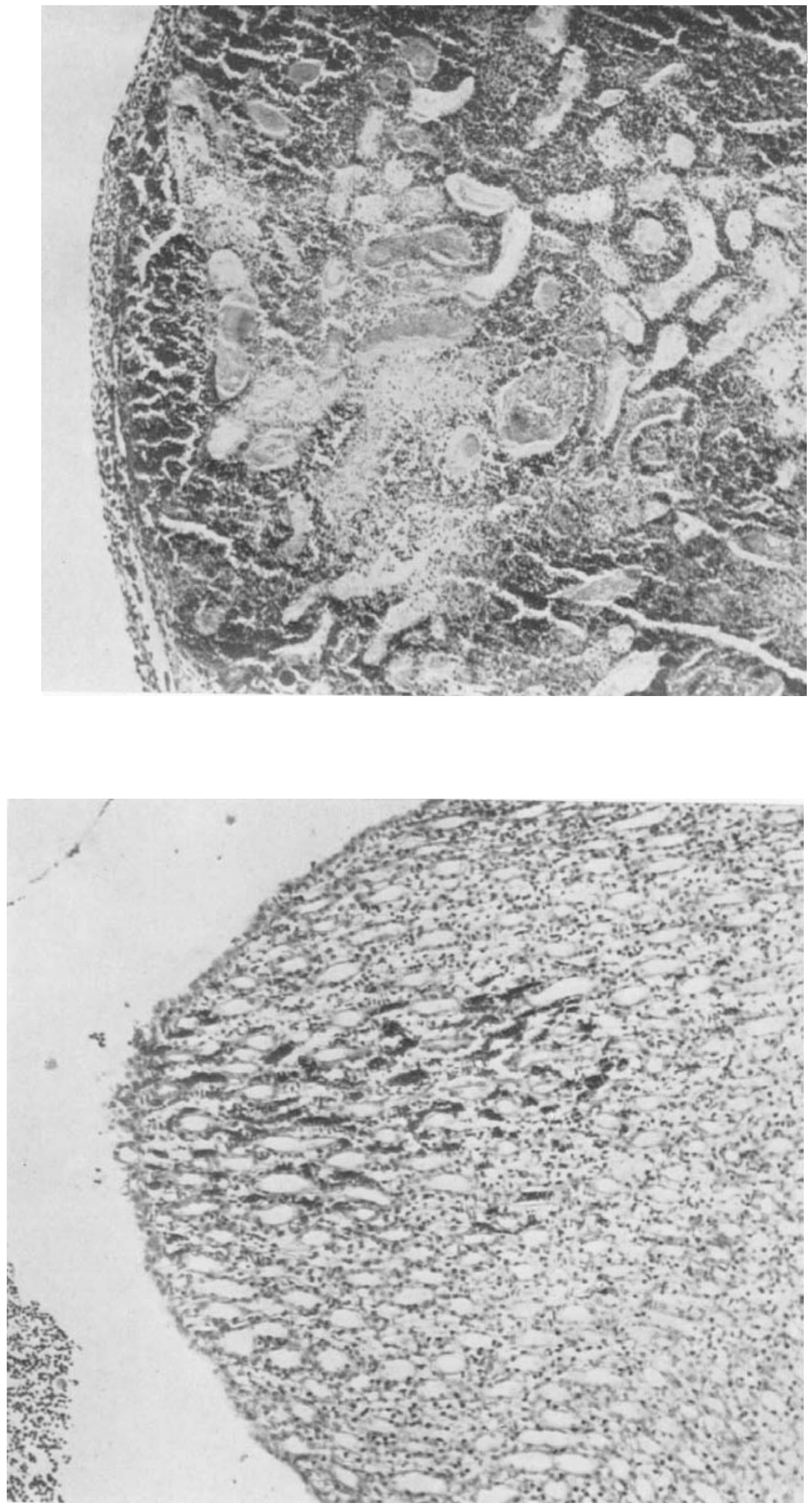